\title{
Evaluation of the Numeric Rating Scale for Perception of Effort during Isometric Elbow Flexion Exercise
}

\author{
Sofia Lampropoulou (1) and Alexander V. Nowicky (2) \\ 1. Physiotherapy Department, Technological Educational Institute of Patras at Aigio, Greece, \\ 25100 , \\ +302691061150 sofia.lampropoulou@yahoo.co.uk
}

2. Centre for Rehabilitation Research, School of Health Sciences and Social Care. Brunel University, Uxbridge, England, UB8 3PH

+4401895268813 Alexander.nowicky@brunel.ac.uk

Final version: 29 June 2011

Corresponding Author: sofia.lampropoulou@yahoo.co.uk

\begin{abstract}
The aim of the study was to examine the reliability and validity of the Numerical Rating Scale (0-10 NRS) for rating perception of effort during isometric elbow flexion in healthy people. 33 individuals ( $32 \pm 8$ years old) participated in the study. Three re-test measurements within one session and three weekly sessions were undertaken to determine the reliability of the scale. The sensitivity of the scale following $10 \mathrm{~min}$ isometric fatiguing exercise of the elbow flexors as well as the correlation of the effort with the electromyographic (EMG) activity of the flexor muscles were tested. Perception of effort was tested during isometric elbow flexion at 10, 30, 50, 70, 90, and 100\% MVC. The 0-10 NRS demonstrated an excellent test-retest reliability (Intra Class Correlation $=0.99$ between measurements taken within a session and 0.96 between 3 consecutive weekly sessions). Exploratory curve fitting for the relationship between effort ratings and voluntary force, and underlying EMG showed that both are best described by power functions $\left(\mathrm{y}=\mathrm{ax}^{\mathrm{b}}\right)$. There were also strong correlations (range 0.89 to 0.95) between effort ratings and EMG recordings of all flexor muscles supporting the concurrent criterion validity of the measure. The 0-10 NRS was sensitive enough to detect changes of the perceived effort following fatigue and significantly increased at the level of voluntary contraction used in its assessment $(\mathrm{p}<0.001)$. These findings suggest the $0-10 \mathrm{NRS}$ is a valid and reliable scale for rating perception of effort in healthy individuals. Future research should seek to establish the validity of the 0-10 NRS in clinical settings.
\end{abstract}

\section{Keywords}

Numeric Rating Scale; Perception of Effort; Scale Sensitivity; EMG; Fatigue; Correlation

\section{Introduction}

Perception of effort during muscular activity is typically defined in a context-dependent manner. It reflects the effort required to complete a task (Noble \& Robertson, 1996) and it has adopted a multidimensional approach with not only physiological (i.e. muscle aches, pain and fatigue) (Bolgar et al., 2010), but also psychological determinants (i.e. task aversion and motivation) (Weiser 1977; Hutchinson and Tenenbaum 2006). Thus, the afferent feedback pathways may play a mediating role in establishing effort intensity. It has been suggested, that the neurophysiological pathway of the perception of effort has its origin in efferent activity of motor commands and that the surface electromyography (sEMG) of the muscles could 
provide an indirect measure of the magnitude of this efferent motor command (Noble \& Robertson, 1996).

Indeed, studies have showed that the perceived effort increases with the level of force produced by the muscle during muscular contractions when participants were asked to estimate their effort (estimation mode of rating) (Jackson \& Dishman, 2000; Pincivero \& Gear, 2000; Rosenbaum \& Gregory, 2002; Pincivero et al., 2003a). During this method of rating, specific force levels were given to participants and they had to estimate the effort that was undertaken to produce these levels of force. A corresponding increase of sEMG activity indicates that the central motor commands compensate for the changes in the peripheral musculoskeletal system. A parallel increment in the perceived effort which was reported by the participants, after effort estimation, suggests that effort is attributable to these motor commands. Indeed, Pincivero and Gear (2000) showed a good correlation not only between force and sEMG activity but also between perceived exertion and sEMG activity. In contrast, when participants were given target level of effort (production mode of rating) and they were asked to match absolute force, an over- production of lower levels of forces and an underproduction of higher level of forces was reported (Pincivero et al., 2003a, b). Thus a force level of 30\% MVC was produced for a level 2 of perceived exertion on a 0-10 rating scale while a force level of $90 \%$ MVC was produced for a level 10 of perceived exertion on a $0-10$ rating scale. An sEMG significantly less than the equivalent ratings of perceived exertion was also reported (Pincivero et al., 2003a, b; West et al., 2005). During the production mode of rating the participants were asked to produce level of forces equivalent to pre-set levels of effort i.e. 2, 3, 4 etc. on a 0-10 rating scale which would be equivalent to $20,30,40 \%$ of Maximum Voluntary Contraction (MVC) accordingly). The overproduction of the low forces could be due to biomechanical characteristics of the lower limb muscles (e.g. knee extensors), which are commonly involved in gross movements related to power and not to accuracy in force reproduction (West et al., 2005). This may be the reason that studies which test accuracy preferentially involve the upper and not the lower limbs for the force production (Carson et al., 2002; Proske et al., 2003; Proske et al., 2004). The underproduction of the higher forces might also be a protective mechanism. In an integrative motor system, there is evidence that the CNS fails to generate maximal force in maximal voluntary efforts and that all motor units in the exercising muscle may never be maximally recruited, even at the onset of a maximal isometric contraction (Gandevia, 2001).

Additionally, during contralateral-limb force matching tasks, fatigue causes an overestimation of the force production and a concurrent increase in the EMG activity of the fatigued muscles indicating increase in the sense of effort (Gandevia \& McCloskey, 1978; Cafarelli \& BiglandRitchie, 1979; Carson et al., 2002). This increased effort required to generate the same muscular force could be explained by the increased voluntary motor activity required to overcome the peripheral and central changes that accompany fatigue (Burgess \& Jones, 1997; Presland et al., 2005).

Various scales have been introduced to assess perceptual experiences in an estimation mode during a physical task. The $0-10$ Numeric rating Scale is one of those which has been extensively used in assessing pain (Kendrick \& Strout, 2005). Among other scales that assess subjective phenomena such as Likert Scales (Grant et al., 1999), Visual Analogue Scale (Crichton, 2001), Numeric Rating Scale (NRS) (Williamson \& Hoggart, 2005), Verbal Rating Scales (VRS) (Lund et al., 2005), the Numeric Rating Scale is more advantageous because: it is easy to administer and score, there are no age-related difficulties in using the scale, it can be delivered either graphically or verbally, and it is an interval level scale and therefore affords parametric statistical testing (Williamson \& Hoggart, 2005). The NRS is an 11 point scale where the end points are the extremes of the subjective or perceived feeling where one end denotes no feeling, and the other, the highest or worst as appropriate (Williamson \& Hoggart, 2005). It has been recommended by the US National Institute of Health as reliable and appropriate for use in clinical and research practice (NIH Pain Consortium). 
The reliability and validity of the 0-10 NRS in assessing pain (Kendrick \& Strout, 2005; Williamson \& Hoggart, 2005) as well as its ease of applicability suggests that a similar methodology could be suitable for effort assessment during brief bouts of isolated muscular activity. The Borg Scale (Ratings of Perceived Exertion (RPE) and the Borg category-ratio 10-item scale are the most common in assessing perceived exertion (Borg, 1998); additionally perceived exertion ratings during isometric contraction have also used these scales (Troianoa et al., 2008); they have greatest prominence is the assessment during dynamic (sustained) aerobic whole body exercise (Gearhart et al., 2001; Dawes et al., 2005; Eston et al., 2005; Kremenic et al., 2009). This is probably due to the high correlation of RPE with heart rate, blood pressure and lactate concentration (Suminski et al., 1997; Capodaglio, 2001, Eston et al., 2005). A scale however, which would be mainly applicable to assess effort during an isometric force production remains to be established.

Therefore, the purpose of this study was to evaluate the 0-10 NRS for assessing perception of effort during elbow flexors isometric muscle action in healthy individuals. Specifically, the 010 NRS was tested for its test-retest reliability and the concurrent criterion validity. In addition, the sensitivity of the scale to detect changes in the effort as the level of voluntary contraction increased as well as following a brief bout of isometric exercise of elbow flexors for the same level of force production has been also examined. For this study we hypothesize that the 0-10 NRS is a valid and reliable measurement tool to assess perception of effort of healthy individuals during isometric elbow flexion and following a fatiguing exercise, and that it is sensitive to changes in perceived effort due to variations in isometric muscle force production as well as due to fatigue caused by the exercise.

\section{Methods}

\section{Sample}

Two experiments were used in this study. The first experiment gave data for the reliability, validity and intensity discrimination of the NRS. These data were gathered from baseline measures repeatedly taken over the same session. Twenty one (14 women and 7 men) healthy volunteers (mean $33 \pm 10$ (SD) years, range: 18 to 63 years) participated in the first experiment. The sensitivity of the scale to detect changes following a brief bout of fatiguing exercise was also tested in the same experiment with a subgroup of 13 participants (10 women and 3 men, $33 \pm 9$ (SD) years, range: 24 to 54 years). During the second experiment the $0-10$ NRS was tested for its reliability and validity between measurements taken over time in three weekly sessions where identical isometric test measurements were undertaken. An additional twelve ( 8 women and 4 men) healthy volunteers (mean age $2 \pm 6(\mathrm{SD})$ years, range: 24 to 42 years) took part in the second experiment. The data set of all the experiments was analyzed using a mixed gender sample and a within-subjects repeated measures design was used. All participants except one were right handed. They were recruited from the university and gave voluntary consent to this study. They were all naïve in using perceptional scales and performing isometric, resistance exercises, and thus they were trained before the experiment. The study was approved by the University Ethics Committee.

\section{Apparatus}

\section{Force Measurements and Surface Electromyography (sEMG)}

Force measurements were obtained from isometric right elbow flexion of all participants using a purpose built static rig containing a force transducer (Model 615, S-Type Load Cell, Tedea-Huntleigh Electronics, UK, force range $\pm 300 \mathrm{~kg}$ ). The analogue force signal was amplified (300 or 1000 times) and filtered (high pass DC-offset, low pass $2 \mathrm{KHz}$ using a signal conditioner (1902, Cambridge Electronic Design (CED), Cambridge, UK). 
sEMG was obtained from $\mathrm{m}$. biceps brachii muscle (BB), $\mathrm{m}$. brachioradialis (BR), and $\mathrm{m}$. brachialis $(\mathrm{Br})$ recorded simultaneously with elbow flexion force. Pairs of silver/silver chloride $(\mathrm{Ag} / \mathrm{AgCl})$ disposable gel recording electrodes (Arbo infant electrodes, circular, $22 \mathrm{~mm}$ in diameter, Henleys Medical Supplies, Herts, UK) were connected in a bipolar, differential configuration (De Luca, 1997). A ground electrode was placed over the medial epicondyle of the humerus. sEMG signals were amplified (1000 or 3000 times), filtered ( $1 \mathrm{~Hz}$ high pass, 2KHz low pass) using a programmable signal conditioner (quad 1902, CED, Cambridge UK). Both the force and sEMG data were digitized (sampling rate of $4 \mathrm{KHz}$ ) using an analogue to digital converter (ADC) (micro 1401, 12 channels, CED, Cambridge, UK) and displayed onscreen during the experiments as well as stored on a PC using software, Spike v.6 for windows (CED, Cambridge UK). Additional off-line analysis of EMG signals and force recordings was undertaken using Signal v.4 for windows (CED, Cambridge UK).

\section{0-10 NRS for Rating Perception of Effort}

The 0-10 NRS was presented graphically during the familiarization session when instructions were given as to its utility (see Fig. 1). This scale uses a horizontal line with each number interval representing an increasing level of effort. The end points are the extremes of "no effort" and the - maximum effort" which could be exerted to produce a maximal voluntary isometric elbow flexion.

\section{$<<<$ Figure 1 about here $>>>$}

\section{Experimental Procedure}

At the beginning of every experimental session due care was given to the correct positioning of the participant (see Fig. 2). An initial familiarization phase (15 minutes) was undertaken when the experimental procedure was explained, and the participants became familiar and comfortable with arm position in use the force rig. Brief trials of isometric contractions of elbow flexion (most of them less than MVC, to avoid fatigue) were attempted to ensure good sustained contractions which had minimal fluctuations around the target force level, and to become familiar with the visual feedback of required force levels. In order to reliably produce a given target force, a horizontal marker line appeared each time and remained on the monitor for visual guidance in order to maintain the required force level. Over the range of force levels, participants were asked to use the rating scale and report the rating of effort of the sustained target level force (see details of effort ratings at perception of effort task). At the end of the familiarization session the participants were rested for ten minutes to limit fatigue.

\section{$<<<$ Figure 2 about here $>>>$}

Once the experimental sessions commenced, participants were asked to undertake the perception of effort task several times according to the protocol. Three perceived effort tasks were performed with a $20 \mathrm{~min}$ time interval before the short bout of fatiguing exercise was undertaken. These measurements gave data for the test-retest reliability analysis of the 0-10 NRS within the same session. Three more perception of effort tasks were performed by the same group of participants after the fatiguing exercise in the same session and these gave data for the sensitivity of the scale to detect changes in the perceived effort for a given level of isometric force production which were detected subsequent to muscle fatigue following a brief bout of fatiguing exercise. In the second experimental study the perceptual tasks were performed over three weekly sessions and the measurements at the same force levels between the sessions were compared to give data for the test-retest reliability analysis between sessions.

\section{Perceived Effort Task}


The perception of effort task is based on an estimation mode of assessment where participants perform contractions without any visual feedback. All target levels of force were based on each participant's maximal voluntary muscle isometric action which was always assessed at the beginning of each session. The MVC was determined as the mean of three, 5 second maximal contractions using strong verbal encouragement which were interspersed with 30 seconds of rest. These maximal attempts were comparable. Participants were prompted to think about the perceived effort during the actual maximal action, and to rate his/her subjective feeling of effort as 10 on the NRS. A point 0 on the NRS, that was explained as remained still and relaxed, corresponded to no effort at all. After they had been reminded that 0 was the lower and 10 was the highest anchor of the $0-10$ point scale, participants were asked to verbally rate the effort that they undertook to initiate and complete a brief sustained $(6 \mathrm{sec})$ voluntary force production. Specific care was given to ask participants about the effort that they exerted to initiate and complete each contraction, and not the degree of difficulty or discomfort caused by the contraction. Ratings were immediately taken at the end of each contraction from all participants, and recorded by the assessor by online keyboard entry of numeric rating. These keyboard entered scores were simultaneously recorded with the data on another input channel and stored with digitized force and EMG data.

Six different levels of voluntary force $(10,30,50,70,90,100 \%$ MVC) were used as target levels of force and presented three times in a randomized manner. The target force level was indicated by a visible horizontal marker line which always appeared in the middle of the screen regardless of the actual level of force and had no visible vertical force (y)-scale. The target marker line ensured visual feedback of force production but without any visual cues to assist in making an assessment of the perceived effort for each trial. The screen was refreshed at the end of each attempt, so that only the current attempt was visible for the given attempt.

The post fatigue effort ratings were obtained at three different levels of voluntary force (10, 30 , and $50 \%$ of pre fatigue MVC) in the same way as described above. During these post fatigue contractions special care was given to ensure that participants reported the effort that they exerted to undertake each contraction, and not the degree of difficulty or discomfort caused by the contraction specifically due to fatigue.

\section{Fatiguing Exercise}

Fatigue was induced by repeated intermittent isometric elbow flexions at 50\% MVC (mean duration of the exercise: $12.5 \pm 3.8 \mathrm{~min}$, range 8 to $15 \mathrm{~min}$ ). Each contraction lasted 15 seconds, and was followed by 2 seconds rest. In pilot work these intervals produced rapid fatigue in a fairly standard and reliable manner. At the beginning and at 3 min intervals during the fatiguing session, participants performed an MVC which was used to determine the degree of fatigue, until these attempts were reduced to $40 \%$ of initial MVC. This reduction in the MVC has been reported previously as a satisfactory indicator for studying fatigue-induced peripheral and central motor changes, and it has been used for assessing central fatigue (Taylor et al., 2000).

\section{Analysis of Data}

The sEMG and the voluntary force were normalized to the maximal values taken from each subject. The amplitude of the surface EMG activity during generation of voluntary force levels was determined by the root mean square (rms) method of analysis for 1.5 second from peak force. The Intra Class Correlation (ICC) (model: ICC2(A,3)) was used for the test retest reliability analysis (Yen \& Lo, 2002) where the -class $2 \|$ indicates that all participants took part at all time points, $-3 \|$ indicates the number of the retest measurements undertaken and $\mathrm{A}$ indicates that the absolute agreement between test and re-test recordings of the NRS for the perception of effort ratings given by the same raters participants) was tested. The ICC 
categories of reliability are as follows: 0.0-0.4: poor, 0.4-0.75: fair to good, and 0.75-1.00: good to excellent (Fleiss, 1986). To assess the criterion validity of the scale, the ratings of the perceived effort were correlated with the objective measurements of the voluntary force produced at every effort rating and with the EMG activity of the muscles participating in the voluntary contractions. Spearman's correlation coefficient was used for the correlations of non-normally distributed data. Changes of the perceived effort with the level of force production as well as effort changes post fatigue (at the same absolute level of force production) and within the same session was assessed with repeated measures analysis of variance (ANOVA), with Bonferroni corrections, (Field, 2005). The Coefficient of Variation (CV) was also calculated, with the root mean square approach, to test variation of the NRS records between subjects. All the statistical tests were performed using SPSS (v.15; for Windows, Chicago: SPSS Inc). Graphs and additional curve-fitting using power functions (y $=\mathrm{ax}^{\mathrm{b}}$ ) were produced using PsiPlot ( $\mathrm{v} 9$ for Windows, Poly software International, New York).

\section{Results}

\section{Force and Perception of Effort}

\section{Accuracy of force production}

An assessment of accuracy of force production - to ensure that all participants although naïve in such kind of exercise were consistent in producing force equal to the target level - was undertaken from the initial trial involving 3 randomized attempts at each of 6 target force levels including the MVC. The within-session ICC for the agreement between target level of force and voluntary force was excellent at every measurement (ICC $=0.99$ (95\% Confidence Interval: $0.98,0.99)$ for the first, ICC $=0.98$ (95\% Confidence Interval: $0.97,0.99)$ for the second, ICC $=0.99$ (95\% Confidence Interval: $0.98,0.99$ ) and for the third trial respectively. The correlation coefficient, between target level of force and the produced voluntary force was also very high for each trial within the session $\left(1^{\text {st }}, \rho=0.981 ; 2^{\text {nd }}, \rho=0.975\right.$; and $3^{\text {rd }}$, $\rho=0.980$, each with $\mathrm{p}<0.001$ ).

\section{Reliability and Variability}

The relationship between NRS0-10 perception of effort ratings and actual force was also determined for the same group of participants $(n=21)$. The ratings of the perceived effort on the NRS increased with the level of produced force (see Table 1). Figure 3 shows the relationship between perception of effort and actual force which was defined by the power equation: $\left.\mathrm{y}=0.271 \mathrm{x}^{0.784}, \mathrm{R}^{2}=0.99\right)$. The within-session ICC, based on the three trials separated by a $20 \mathrm{~min}$ intervals, for the rating of the perceived effort was 0.99 (95\% Confidence Interval (CI) $0.98,0.99)$ indicating that the participants were consistent in rating effort for the same level of force production. The between-session ICC, based on the three trials separated by the week intervals, for perception of effort and force also revealed excellent reliability (ICC $=0.96(95 \% \mathrm{CI}=0.96,0.97)$. No significant differences in the effort ratings were revealed from the $1^{\text {st }}$ to $2^{\text {nd }}$ session separated by a week $\left(\mathrm{F}_{(2,20)}=0.31, p=0.74\right.$, Partial Eta Squared $=0.03$ ). The variability of the measurements taken by the $0-10$ NRS was low; $17 \%$ for the measurements taken with a week interval and only $6 \%$ for those taken within a session.

$<<$ Table 1 about here $\gg \gg>$

$<<<$ Figure 3 about here $\gg>>>$

Perception of Effort and Surface Electromyography 
The perception of effort increased with sEMG of the three elbow flexor muscles, as it was normalized to \% MVC in session 1 (see Table 1). The relationship between the perception of effort and the rms EMG level of activation for the indicative muscle, $\mathrm{m}$. biceps brachii, was also modeled with a power function $y=0.733 x^{0.558}, R^{2}=0.99$ (see Figure 4). A significant main effect of the intensity of voluntary contraction on $\mathrm{SEMG}$ of $\mathrm{BB}, \mathrm{Br}$ and $\mathrm{BR}$ was also revealed (for BB: $\mathrm{F}_{(1.82,36.46)}=493.77, p<0.001$, Partial Eta Squared $=0.96$, for $\mathrm{Br}: \mathrm{F}_{(1.76 \text {, }}$ ${ }_{35.17)}=625.20, p<0.001$, Partial Eta Squared=0.97, for BR: $\mathrm{F}_{(2.51,50.19)}=795.85, p<0.001$, Partial Eta Squared=0.98). For example, for lowest level of force production (10\%MVC), BB EMG was $8 \pm 3.4(\mathrm{SD}) \% \mathrm{Max}$ and increased to $104 \pm 16$ (SD) \%Max at $100 \%$ MVC. All correlation coefficients between effort and EMG or voluntary force were above 0.89 (range 0.89 to 0.95 ) for the three trials taken within the same session. Similarly correlations were above 0.86 (range 0.86 to 0.93 ) when the measures of force, effort and normalized sEMG were repeated in three sessions separated by weekly intervals.

\section{$<<<$ Figure 4 about here $\gg \gg$}

\section{Sensitivity of the 0-10 NRS}

\section{Different levels of voluntary force production}

Perception of effort was significantly increased with the level of voluntary contraction $\left(\mathrm{F}_{(2.23}\right.$, $44.66=805.70, \mathrm{p}<0.001$, Partial Eta Squared=0.98). Pairwise comparisons revealed that the effort ratings at every level of force production were significantly higher than the ratings at the preceding level $(p<0.001)$. Table 4 presents the differences in the NRS ratings between levels of voluntary contraction. The smallest difference presented in the table to be statistically significant was a difference of 0.67 categories on the NRS.

\section{$<<$ Table 2 about here $\gg \gg>$}

\section{Following the Fatiguing Exercise}

Ten minutes of intermittent isometric elbow flexion at 50\% of MVC caused a $40 \%$ drop of the MVC which was significant, (mean difference $60 \pm 7$ (SE) N, $p<0.001$ ). Despite some recovery in the MVC force production following the fatiguing exercise, the MVC remained significantly reduced at post 60 minutes (mean difference $19 \pm 4(\mathrm{SE}) \mathrm{N}, p=0.008$ ). Perceived effort increased significantly following the fatiguing exercise $\left(\mathrm{F}_{(5,65)}=22.97, \mathrm{p}<0.001\right.$, Partial Eta Squared=0.64). An increase of 1 category on the NRS for the low level of contraction and 2 categories in the rating of the perceived effort on the NRS for contractions at 30 and $50 \%$ of MVC was observed (see Figure. 5). Specifically, the mean increase of the perceived effort among all the levels of voluntary force was 1.6 categories on the NRS 10 minutes post fatiguing exercise (mean difference $=1.64 \pm 0.20$ (SEM), $p<0.001,95 \% \mathrm{CI}=1.03$ to 2.25 ) over baseline. The increase in perception of effort was still significant at thirty minutes (mean difference $=1.24 \pm 0.18$ (SEM), $p<0.001,95 \% \mathrm{CI}=0.67$ to 1.81 ), and at 50 minutes (mean difference $=1.03 \pm 0.23$ (SEM), $p=0.004,95 \% \mathrm{CI}=0.31$ to 1.76 ). With the increase in effort there was a corresponding increase in the mean sEMG in all muscles: $\mathrm{BB}\left(\mathrm{F}_{(1.67,1.75)}=7.59\right.$, $\mathrm{p}<0.001$, Partial Eta Squared $=0.37)$, of $\operatorname{Br}\left(\mathrm{F}_{(1.97,25.63)}=14.83, \mathrm{p}<0.001\right.$, Partial Eta Squared $=0.53)$ and $B R\left(F_{(3,39)}=11.68, p<0.001\right.$, Partial Eta Squared $\left.=0.47\right)$.

\section{$<<<$ Figure 5 about here $\gg>>>$}

\section{Discussion}

The $0-10$ NRS has been assessed for its reliability and validity in ratings of the perceived effort under various levels of isometric elbow flexion. The test-retest reliability of the $0-10$ NRS was excellent both within a single session and between three consecutive weekly 
sessions. Indeed, perceived effort, as it was reported on the NRS, significantly increased with the intensity of the voluntary contraction while the levels of perceived effort matched \%MVC target force intensities. The significant correlation of the effort ratings with the sEMG activity of all flexor muscles suggests that this method of measurement has good concurrent criterion validity. Additionally, the scale is sensitive in recording changes in the perception of effort with force production levels, as well as to the effort increase following fatigue. It may therefore reflect the changing physiological demands of this short duration fatiguing isometric exercise protocol. These findings support the research hypothesis that the $0-10$ NRS is a reliable and valid method of recording perception of effort for isolated isometric muscle action.

The excellent test-retest reliability (0.99) of the $0-10$ NRS indicates that $99 \%$ of the variance in the ratings results from - truell variance among subjects and not from measurement error (Streiner \& Norman, 2003). The small variance, as well as the consistency of the ratings, not only within the same session measurements, but also in a series of weekly separated trials indicates its reliability and ease to use. However, the reliability of the NRS may be due to the relatively short interval between the test and retest protocol used here, and should be tested over longer periods. This short interval within a session and between weeks, might lead to a learning effect where there may be recognition of repeated trial intensities (Williamson \& Hoggart, 2005). However, this is more likely to occur when only a small number of levels are used. In the present study, six different levels of voluntary contractions were applied, taken from the full available range of contractions, with three repeated random presentations, which minimized response demand bias.

A power function provided the best fit for the relationship between effort rating and force production for isolate isometric exercise used in this study. Similarly, the relationship between effort rating and underlying isometric elbow sEMG activity over range of produced voluntary forces was also described by a power function. It has been suggested that psychological components of the perceptual effort reports reflecting motivation and affect, could contribute to this nonlinear relationship (Hutchinson \& Tenenbaum 2006). In addition, physical changes induced by muscle fatigue and aches due to exercise may contribute to the nonlinear force-effort relationship. It has been suggested in pain assessment studies where moderate pain is reached, relatively small increases in stimulation may result in exponential (nonlinear) enhancement of pain report (Janal, 1995, cited in Hartrick et al., 2003). The same could be applied to the effort ratings, although pain and effort are different subjective feelings that are perceived under different processes.

The present study has also revealed an overestimation at the moderate levels of force production, meaning that the participants rated their effort to produce a given level of force more than the equivalent level of effort. The involvement of the upper arm in everyday tasks at moderate levels of force workload may be the reason for the overestimation at these levels. The studies of Pincivero (2003a, b) and West (2005) have also reported a perceptual overestimation at moderate to high levels of voluntary contraction and an underestimation of effort at nearly maximum levels of voluntary contraction. However, comparisons with these studies may not be appropriate because the data of these studies are based on a production mode of assessment while our data are derived under estimation mode of assessment. Additionally the CR10 Borg scale has been used for perceptually guided contractions. The Borg scale however, has intermediate anchors (Dawes et al., 2005) and an opened top anchor which allows for ratings higher than expected according to previous experiences (Borg 1998) and as such would allow for difference in the perceptual recordings.

Additionally, although the establishment of the validity of the 0-10 NRS has not been based on the relationships of the NRS ratings to other tools that measure the same construct, the constant associations of the NRS ratings with the voluntary force and the underlying sEMG activity provide further evidence to support the concurrent criterion validity of the $0-10$ NRS. 
It is difficult to measure perception of effort directly because invariably it is a complex process involving many areas in the central nervous system in addition to primary sensorimotor activity (McCloskey, 1981). The strong association of the NRS with the sEMG when the intensity of the voluntary contraction increases, as well as under fatiguing conditions, supports the assumption that perception of effort is primarily an efferent mechanism that involves higher CNS centers. The efferent signal is proportional to the magnitude of voluntary motor command and is likely mediated by afferent inputs (Gandevia, 2001). The changes in EMG activity of the muscles during an exercise of increased intensity, indicates increased central drive to the muscles through recruitment of more motor units. Thus, when the demands in the periphery increase due to enhanced workload or fatigue, the increased EMG activity is followed by an increase in the perceived effort (Liu et al., 2003). This may be explained by the feedforward-feedback system where perception of effort does change whenever there is a mismatch between the corollary discharges that radiate to the somatosensory cortex and the afferent impulses evoked in the periphery as result of the motor command (Wallman \& Sacco, 2007). Implementation of the 0-10 NRS with the use of EMG therefore, adds information about the neurophysiological basis of the perception of effort and point towards a measurement tool that is able to follow neurophysiological alterations due to exercise.

A statistically significant change of 0.7 categories on the scale indicates a sensitive tool that could detect changes in perception of effort of even a small absolute size. The changes in the perception of effort that were detected by the 0-10 NRS following fatigue further support the potential applicability of the scale for assessing changes in perception of effort following an intervention.

The ability of the 0-10 NRS to detect changes in the effort when isolated muscles are exercised and its ability to follow the neurophysiological alterations caused by the isometric exercise supports its potential applicability in assessing perception of effort whenever the whole body activation is restricted. Further testing is required of this scale in the healthy population where greater control of sample selection with respect to age and gender related differences could be examined.

In conclusion, the 0-10 NRS demonstrated an excellent test-retest reliability and good concurrent criterion validity in recording perception of effort under repeated isometric contractions of elbow flexors. The power function of the effort ratings on the 0-10 NRS with the level of force production and the sEMG as well as the significant changes that were revealed following the fatiguing exercise suggest that this is sensitive to neurophysiological alterations due to isometric exercise. As the validation of the NRS for the perception ratings has been confined to a healthy population, the effectiveness and applicability of the effort NRS within the clinical field has yet to be explored.

\section{Ethical standards}

The experiments have received ethical approval from Brunel University Ethics Committee.

\section{Conflict of Interest}

The authors declare that they have no conflict of interest.

\section{Acknowledgments}

The authors would like to acknowledge the financial support of the School of Health Science and Social Care of Brunel University, and to thank staff and student participants of the university who volunteered in this study. 


\section{References}

1. Bolgar MR, Baker CE, Goss FL, Nagle E, Robertson RJ. Effect of exercise intensity on differentiated and undifferentiated ratings of perceived exertion during cycle and treadmill exercise in recreationally active and trained women, Journal of Sports Science and Medicine, 2010; 9:557-563.

2. Borg G. Borg's Perceived Exertion and Pain Scales, USA, Human Kinetics, 1998.

3. Breivik EK, Bjornsson GA, Skovlund E. A comparison of pain rating scales by sampling from clinical trial data, The Clinical Journal of Pain, 2000; 16(1):22-28.

4. Burgess PR, Jones LF. Perceptions of effort and heaviness during fatigue and during the size-weight illusion, Somatosensory \& motor research, 1997; 14(3):189-202.

5. Cafarelli E, Bigland-Ritchie B. Sensation of static force in muscles of different length, Experimental Neurology, 1979; 65(3):511-525.

6. Capodaglio EM. Comparison between the CR10 Borg's scale and the VAS (visual analogue scale) during an arm-cranking exercise, Journal of Occupational Rehabilitation, 2001; 11(2):69-74.

7. Carson RG, Riek S, Shahbazpour N. Central and peripheral mediation of human force sensation following eccentric or concentric contractions, The Journal of Physiology, 2002; 539(Pt 3):913-925.

8. Crichton N. Visual Analogue Scale (VAS), Blackwell Science Ltd, Journal of Clinical Nursing, 10, 697士706, 2001; 10(5):706.

9. Dawes HN., Barker KL, Cockburn J, Roach N, Scott O, Wade D. Borg's rating of perceived exertion scales: do the verbal anchors mean the same for different clinical groups? Archives of Physical Medicine and Rehabilitation, 2005; 86(5): 912-916.

10. De Luca CJ. The use of surface electromyography in biomechanics, Journal of Applied Biomechanics, 1997; 13:135-163.

11. Eston RG, Lamb KL, Parfitt G. The validity of predicting maximal oxygen uptake from a perceptually- regulated graded exercise test, European Journal of Applied Physiology, 2005; 94:221-227.

12. Field E. Discovering Statistics Using SPSS, 2nd ed., London, Thousand Oaks, New Delhi, SAGE Publications, 2005.

13. Gandevia SC. Spinal and supraspinal factors in human muscle fatigue, Physiological Reviews, 2001; 81(4):1725-1789.

14. Gandevia SC, McCloskey DI. Interpretation of perceived motor commands by reference to afferent signals, The Journal of Physiology, 1978; 283:493-499.

15. Gearhart RE, Goss FL, Lagally KM, Jakicic JM, Gallagher J, Robertson RJ. Standardized scaling procedures for rating perceived exertion during resistance exercise, Journal of Strength and Conditioning Research / National Strength \& Conditioning Association, 2001; 15(3):320-325.

16. Grant S, Aitchison T, Henderson E, Christie J, Zare S, McMurray J, et al. A comparison of the reproducibility and the sensitivity to change of visual analogue scales, Borg scales, and Likert scales in normal subjects during submaximal exercise, Chest, 1999; 116(5):1208-1217. 17. Hartrick CT, Kovan JP, Shapiro S. The numeric rating scale for clinical pain measurement: a ratio measure? Pain Practice, 2003; 3(4): 310-316.

18. Hutchinson JC, Tenenbaum G. Perceived effort — Can it be considered gestalt? Psychology of Sport and Exercise, 2006; 7:463-476.

19. Kendrick DB, Strout TD. The minimum clinically significant difference in patient assigned numeric scores for pain, The American Journal of Emergency Medicine, 2005; 23(7):828-832.

20. Kremenic IJ, Glace BW, Ben-Avi SS, Nicholas SJ, McHugh MP. Central fatigue after cycling evaluated using peripheral magnetic stimulation. Medicine of Science in Sports \& Exercise. 2009; 41(7):1461-6.

21. Koppelaar E, Wells R. Comparison of measurement methods for quantifying hand force, Ergonomics, 2005; 48(8):983-1007. 
22. Liu JZ, Shan ZY, Zhang LD, Sahgal V, Brown RW, Yue GH. Human brain activation during sustained and intermittent submaximal fatigue muscle contractions: an FMRI study, Journal of Neurophysiology, 2003; 90(1):300-312.

23. Lund I, Lundeberg T, Sandberg L, Budh CN, Kowalski J, Svensson E. Lack of interchangeability between visual analogue and verbal rating pain scales: a cross sectional description of pain aetiology groups, BMC Medical Research Methodology, 2005; 5:31 24. NIH Pain Consortium, pain intensity scales. Available from: http://painconsortium.nih.gov/pain_scales/index.html. 25. Noakes TD, St Clair Gibson A, Lambert EV. From catastrophe to complexity: a novel model of integrative central neural regulation of effort and fatigue during exercise in humans: summary and conclusions, British Journal of Sports Medicine, 2005; 39(2):120-124.

26. McCloskey DI. Corollary Discharge: Motor Commands and Perception, in: Brooks VB, (ed), Handbook of Physiology. The Nervous System, Motor Control, Bethesda, MD, American Physiological Society, 1981. 1415-1447 p.

27. Pincivero DM, Coelho AJ, Campy RM, Salfetnikov Y, Suter E. Knee extensor torque and quadriceps femoris EMG during perceptually-guided isometric contractions, Journal of Electromyography and Kinesiology, 2003a; 13(2):159-167.

28. Pincivero DM, Dixon PT, Coelho AJ. Knee extensor torque, work, and EMG during subjectively graded dynamic contractions, Muscle \& Nerve, 2003b; 28(1):54-61.

29. Pincivero DM, Gear WS. Quadriceps activation and perceived exertion during a high intensity, steady state contraction to failure, Muscle \& Nerve, 2000; 23(4):514-520.

30. Presland J.D, Dowson MN, Cairns SP. Changes of motor drive, cortical arousal and perceived exertion following prolonged cycling to exhaustion, European Journal of Applied Physiology, 2005; 95(1):42-51.

31. Proske U, Weerakkody NS, Percival P, Morgan DL, Gregory JE, Canny BJ.

Forcematching errors after eccentric exercise attributed to muscle soreness,

Clin.Exp.Pharmacol.Physiol., 2003; 30(8):576-579.

32. Proske U, Gregory JE, Morgan DL, Percival P, Weerakkody NS, Canny BJ. Force matching errors following eccentric exercise, Hum.Mov.Sci., 2004; 23(3-4):365-378.

33. Slobounov S, Hallett M, Newell KM. Perceived effort in force production as reflected in motor-related cortical potentials, Clinical Neurophysiology, 2004; 115(10):2391-2402.

34. Streiner DL, Norman GR. Reliability, in: Streiner D.L., and Norman, G.R., (eds), Health Measurement Scales: A Practical Guide to their Development and Use, 3rd ed., New York, Oxford University Press, 2003. 137-144 p.

35. Suminski RR, Robertson RJ, Arslanian S, Kang J, Utter AC, DaSilva SG, et al. Perception of Effort During Resistance Exercise, Journal of Strength \& Conditioning Research, 1997; 11(4):261-265.

36. Taylor JL, Butler JE, Gandevia SC. Changes in muscle afferents, motoneurons and motor drive during muscle fatigue, European Journal of Applied Physiology, 2000; 83(2-3):106115.

37. Troianoa A, Naddeoa F, Sossoa E, Camarotab G, Merlettia R, Mesina L. Assessment of force and fatigue in isometric contractions of the upper trapezius muscle by surface EMG signal and perceived exertion scale, Gait \& Posture, 2008; 28 (2):179-186.

38. Wallman KE, Sacco P. Sense of effort during a fatiguing exercise protocol in chronic fatigue syndrome, Research in Sports Medicine, 2007; 15(1):47-59.

39. Weerakkody N, Percival P, Morgan DL, Gregory JE, Proske U. Matching different levels of isometric torque in elbow flexor muscles after eccentric exercise, Experimental brain research, 2003; 149(2):141-150.

40. Weiser PC, and Stamper DA. Psychophysiological interactions leading to increased effort, leg fatigue, and respiratory distress during prolonged, strenuous bicycle riding, in: Physical work and effort, ed: Borg G, New York, Pergamon Press, 1977; 401-416.

41. West SJ, Smith L, Lambert EV, Noakes TD, St Clair Gibson A. Submaximal force production during perceptually guided isometric exercise, European Journal of Applied Physiology, 2005; 95(5-6):537-542. 
42. Williamson A, Hoggart B. Pain: a review of three commonly used pain rating scales, Journal of Clinical Nursing, 2005; 14(7):798-804.

43. Yen M, Lo LH. Examining test-retest reliability: an intra-class correlation approach, Nursing Research, 2002; 51(1):59-62. 


\section{Tables}

Table 1

Table 1: Mean Group $( \pm \mathrm{SD})$ actual voluntary force, ratings of perceived effort, normalized sEMG for elbow flexors: $\mathrm{Br}, \mathrm{BB}, \mathrm{BR}(\mathrm{n}=21)$ at target levels of force $(10,3050,70,90,100 \% \mathrm{MVC})$. The measurements were taken during the baselines before the fatiguing exercise. Three attempts were performed by every participant at every target level of force.

\begin{tabular}{ccccc}
\hline \hline $\begin{array}{l}\text { Voluntary } \\
\text { Force }(\% M V C)\end{array}$ & $\begin{array}{c}\text { Effort } \\
(\text { NRS rating) }\end{array}$ & $\begin{array}{l}\text { Br rmsEMG } \\
(\% M a x)\end{array}$ & $\begin{array}{l}\text { BB rmsEMG } \\
(\% M a x)\end{array}$ & $\begin{array}{l}\text { BR rmsEMG } \\
(\% M a x)\end{array}$ \\
\hline $14.51 \pm 3.02$ & $1.84 \pm 0.73$ & $10.60 \pm 4.32$ & $8.10 \pm 3.42$ & $6.25 \pm 2.90$ \\
$34.18 \pm 2.97$ & $4.28 \pm 0.82$ & $24.00 \pm 5.78$ & $21.16 \pm 4.89$ & $19.97 \pm 5.63$ \\
$53.57 \pm 2.83$ & $6.17 \pm 0.81$ & $44.66 \pm 7.06$ & $41.49 \pm 8.15$ & $42.86 \pm 9.50$ \\
$72.70 \pm 2.82$ & $7.81 \pm 0.52$ & $72.44 \pm 8.08$ & $69.48 \pm 10.38$ & $73.60 \pm 11.23$ \\
$90.63 \pm 2.79$ & $9.16 \pm 0.47$ & $95.38 \pm 9.62$ & $94.77 \pm 13.34$ & $94.85 \pm 10.70$ \\
$97.09 \pm 3.95$ & $9.83 \pm 0.25$ & $105.73 \pm 12.00$ & $104.10 \pm 16.01$ & $100.86 \pm 10.51$ \\
\hline
\end{tabular}


Table 2

Table 2: Ratings of perceived effort (mean difference \pm SD of the effort ratings on the NRS) among levels of force production during baseline measurement $(n=21)$.

\begin{tabular}{|c|c|c|c|c|c|}
\hline \multicolumn{2}{|c|}{ Level of Force (\%MVC) } & \multirow{2}{*}{$\begin{array}{c}\text { Mean Difference (2-1) } \\
(\text { Mean } \pm \text { SEM) }\end{array}$} & \multirow[b]{2}{*}{ Sig. } & \multicolumn{2}{|c|}{ 95\% Confidence Interval } \\
\hline Level 1 & Level 2 & & & Lower Bound & Upper Bound \\
\hline 10 & 30 & $2.44 \pm 0.12$ & $0.001 *$ & 2.83 & 2.04 \\
\hline 30 & 50 & $1.89 \pm 0.10$ & $0.001 *$ & 2.23 & 1.55 \\
\hline 50 & 70 & $1.64 \pm 0.13$ & $0.001 *$ & 2.08 & 1.20 \\
\hline 70 & 90 & $1.35 \pm 0.09$ & $0.001 *$ & 1.65 & 1.05 \\
\hline 90 & 100 & $0.67 \pm 0.08$ & $0.001 *$ & 0.92 & 0.41 \\
\hline
\end{tabular}




\title{
Legends of Figures
}

\author{
Figure 1
}

Fig. 1 0-10 Numeric Rating Scale used to assess perception of effort. Effort was rated as whole number between the two anchors: 0 relaxed with no effort, and 10 during maximum voluntary contraction (MVC).

Figure 2

Fig. 2 Rig for measurement of isometric elbow force used in the study. Participants were seated with right arm resting on an inclined platform, with shoulder flexed slightly $\left(15^{\circ}\right)$ and abducted, with $90^{\circ}$ of elbow flexion. The forearm rested in a semi rigid, felt lined splint in semi-supination, with dense foam padding underneath elbow and forearm. The transducer was secured to a metal plate combined with a flattened-concave plastic block lined with foam so that upward force of elbow flexion could be measured. Transducer-wrist block array was lowered and positioned against wrist with slight downward force $(<2 \mathrm{~N})$ to ensure comfortable and secure fit. Once positioned, the transducer-wrist block locked into place by retaining screws on each of the vertical support tower. Visual feedback of the force recording was provided on a 19 inch LCD monitor positioned in front of the participant. The participant remained in the rig for the duration of an experiment, but if needed, the arm could be removed and repositioned. Pairs of electrodes were affixed over muscles of elbow flexion as shown to for surface electromyography.

Figure 3

Fig 3 Relationship between the perception of effort as it was recorded with the 0-10NRS and the voluntary force levels. Three attempts were performed at every target level of force production $(10,30,50,70,90,100 \%$ MVC). The relationship was characterized by a power function (Fitting model: $\mathrm{y}=\mathrm{ax}{ }^{\mathrm{b}}$, where $\mathrm{a}=0.27$ and $\left.\mathrm{b}=0.784, \mathrm{R}^{2}=0.99\right)$. Results are group mean $\pm \mathrm{SEM}(\mathrm{n}=21)$.

\section{Figure 4}

Fig. 4 The relationship between the perception of effort recorded with the 0-10NRS and the normalized (\%MVC) $\mathrm{m}$. biceps brachii sEMG activity during the baseline measurements before fatigue. Three attempts were performed at every target level of force production $(10,30,50,70,90$, $100 \%$ MVC). Fitting Model: $\mathrm{y}=\mathrm{ax}^{\mathrm{b}}, \mathrm{a}=0.733, \mathrm{~b}=0.558, \mathrm{R}^{2}=0.99$. Data represent group mean \pm SEM $(n=21)$. 


\section{Figure 5}

Fig. 5 Perceived Effort changes during the post fatigue recovery period at voluntary contractions of 30,50 and $70 \%$ of pre fatigue MVC. The effort ratings were significantly increased post fatigue compared to pre fatigue (pre) measurements. Despite some reduction in the effort ratings, which was revealed after the first 10 minutes post the fatiguing exercise, these remained significantly higher than the ratings at baseline measurements. Group mean \pm SEM, $n=13$. Asterisks indicate significant increase in the effort ratings post fatigue (at all time points) when they were compared to pre fatigue ratings (pre). 
Figure 1

\begin{tabular}{|c|c|c|c|c|c|c|c|c|c|c|}
\hline No effort & & & & & & & & & & Max Effort \\
\hline 0 & 1 & 2 & 3 & 4 & 5 & 6 & 7 & 8 & 9 & 10 \\
\hline
\end{tabular}

Fig 1. 0-10 Numeric Rating Scale used to assess perception of effort. Effort was rated as whole number between the two anchors: 0 relaxed with no effort, and 10 during maximum voluntary contraction (MVC). 
Figure 2

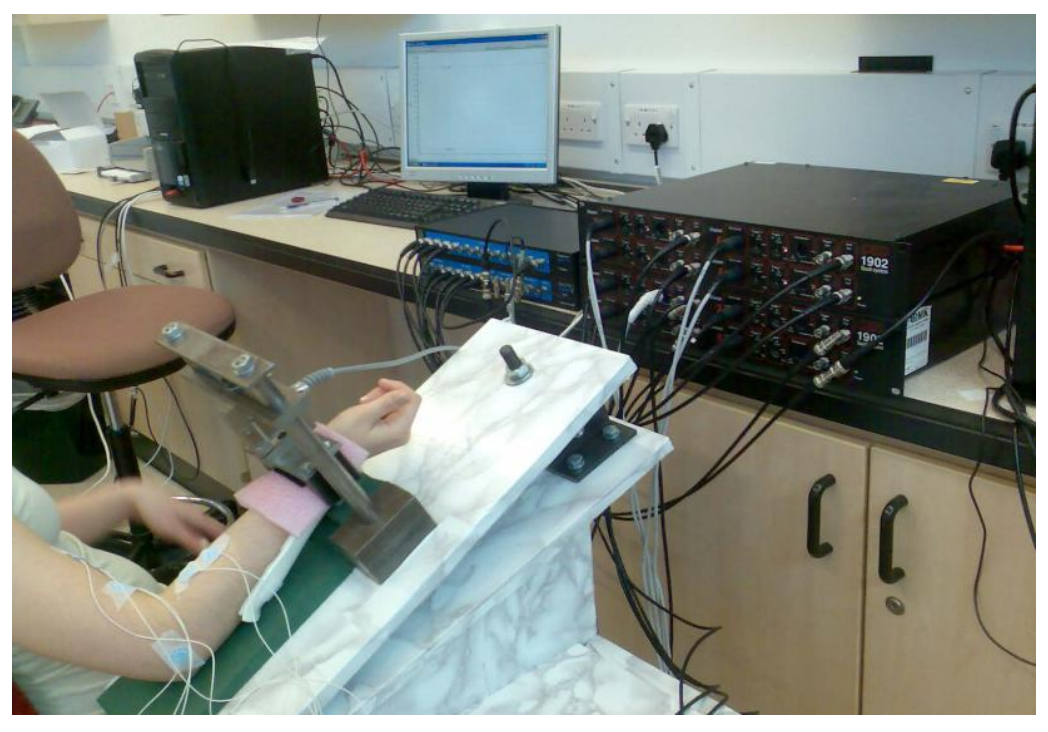

Fig. 2 Rig for measurement of isometric elbow force used in the study. Participants were seated with right arm resting on an inclined platform, with shoulder flexed slightly $\left(15^{0}\right)$ and abducted, with $90^{\circ}$ of elbow flexion. The forearm rested in a semi rigid, felt lined splint in semi-supination, with dense foam padding underneath elbow and forearm. The transducer was secured to a metal plate combined with a flattened-concave plastic block lined with foam so that upward force of elbow flexion could be measured. Transducer-wrist block array was lowered and positioned against wrist with slight downward force $(<2 \mathrm{~N})$ to ensure comfortable and secure fit. Once positioned, the transducer-wrist block locked into place by retaining screws on each of the vertical support tower. Visual feedback of the force recording was provided on a 19 inch LCD monitor positioned in front of the participant. The participant remained in the rig for the duration of an experiment, but if needed, the arm could be removed and repositioned. Pairs of electrodes were affixed over muscles of elbow flexion as shown to for surface electromyography. 


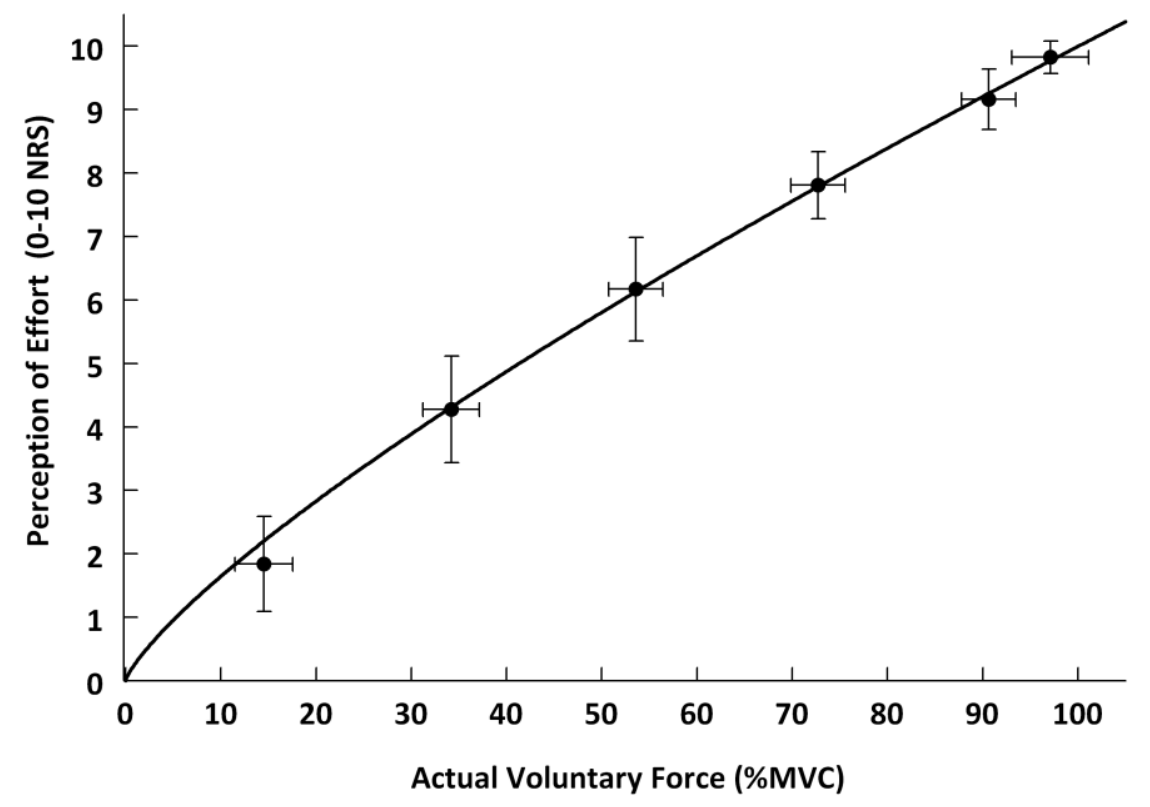

Fig. 3 Relationship between the perception of effort as it was recorded with the 0-10NRS and the voluntary force levels. Three attempts were performed at every target level of force production $(10,30,50,70,90,100 \% \mathrm{MVC})$. The relationship was characterized by a power function (Fitting model: $y=a x^{b}$, where $a=0.27$ and $b=0.784, R^{2}=0.99$ ). Results are group mean \pm SEM $(n=21)$. 


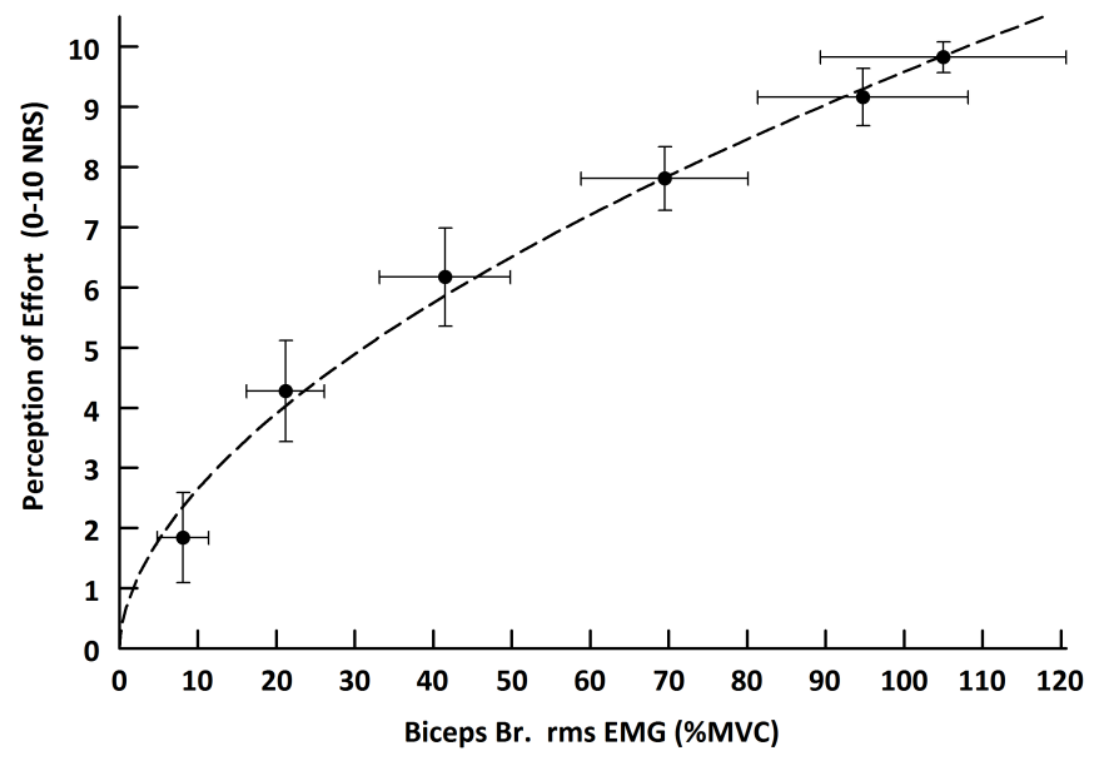

Fig. 4 The relationship between the perception of effort recorded with the 0-10NRS and the normalized (\%MVC) $\mathrm{m}$. biceps brachii sEMG activity during the baseline measurements before fatigue. Three attempts were performed at every target level of force production $(10,30,50,70$, $90,100 \%$ MVC). Fitting Model: $\mathrm{y}=\mathrm{ax}{ }^{\mathrm{b}}, \mathrm{a}=0.733, \mathrm{~b}=0.558, \mathrm{R}^{2}=0.99$. Data represent group mean \pm $\operatorname{SEM}(\mathrm{n}=21)$. 
Figure 5

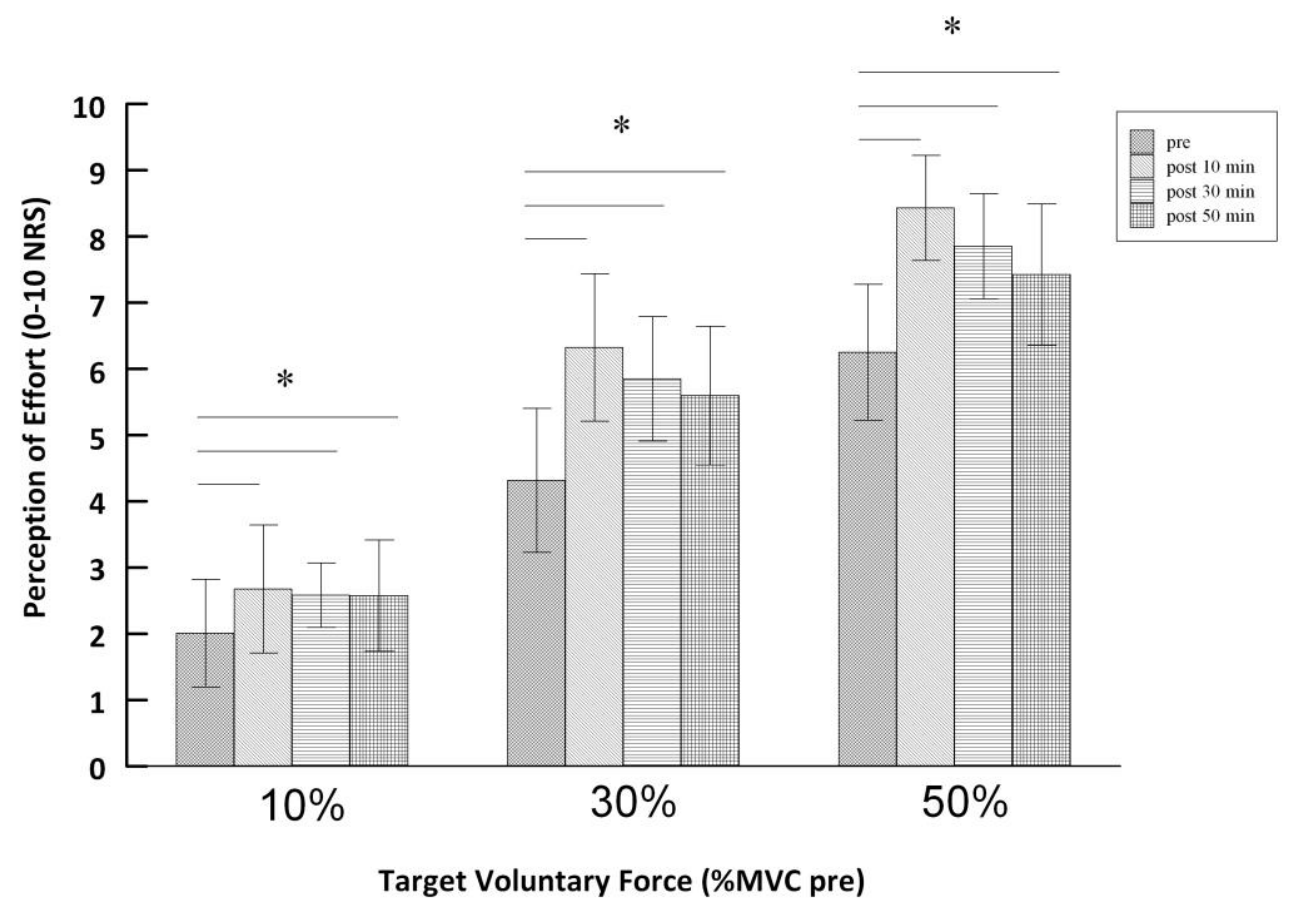

Fig. 5 Perceived Effort changes during the post fatigue recovery period at voluntary contractions of 30, 50 and $70 \%$ of pre fatigue MVC. The effort ratings were significantly increased post fatigue compared to pre fatigue (pre) measurements. Despite some reduction in the effort ratings, which was revealed after the first 10 minutes post the fatiguing exercise, these remained significantly higher than the ratings at baseline measurements. Group mean \pm $\mathrm{SEM}, \mathrm{n}=13$. Asterisks indicate significant increase in the effort ratings post fatigue (at all time points) when they were compared to pre fatigue ratings (pre). 